\title{
Construction of wild-type Yarrowia lipolytica IMUFRJ 50682 auxotrophic mutants using dual CRISPR/Cas9 strategy for novel biotechnological approaches
}

\author{
Camilla Pires de Souza ${ }^{\mathrm{a}, \mathrm{b}}$, Bernardo Dias Ribeiro ${ }^{\mathrm{b}}$, Maria Alice Zarur Coelho ${ }^{\mathrm{b},{ }^{*} *}$, \\ Rodrigo Volcan Almeida ${ }^{\mathrm{a}, *}$, Jean-Marc Nicaud ${ }^{\mathrm{c}, * * *}$ \\ ${ }^{a}$ Department of Biochemistry, Institute of Chemistry, Federal University of Rio de Janeiro, Rio de Janeiro, 21941-909, RJ, Brazil \\ ${ }^{\mathrm{b}}$ Biochemical Engineering Department, School of Chemistry, Federal University of Rio de Janeiro, Rio de Janeiro, 21941-909, RJ, Brazil \\ ${ }^{\mathrm{c}}$ Micalis Institute, INRAE, AgroParisTech, Université Paris-Saclay, 78350 Jouy-en-Josas, France
}

\section{A R T I C L E I N F O}

\section{Keywords:}

Yarrowia lipolytica Brazilian strain

URA3 gene disruption

Dual cleavage CRISPR/Cas9-mediated

Biotransformation

Metabolic engineering

Carotenoid production

\begin{abstract}
A B S T R A C T
Yarrowia lipolytica IMUFRJ 50682 is a Brazilian wild-type strain with potential application in bioconversion processes which can be improved through synthetic biology. In this study, we focused on a combinatorial dual cleavage CRISPR/Cas9-mediated for construction of irreversible auxotrophic mutants IMUFRJ 50682, which genomic information is not available, thought paired sgRNAs targeting upstream and downstream sites of $U R A 3$ gene. The disruption efficiency ranged from 5 to $28 \%$ for sgRNAs combinations closer to URA3's start and stop codon and the auxotrophic mutants lost about $970 \mathrm{bp}$ containing all coding sequence, validating this method for genomic edition of wild-type strains. In addition, we introduced a fluorescent phenotype and achieved cloning rates varying from 80 to $100 \%$. The ura3s strains IMUFRJ 50682 were also engineered for $\beta$-carotene synthesis as proof of concept. Carotenoid-producing strains exhibited a similar growth profile compared to the wild-type strain and were able to synthesized 30.54-50.06 mg/L (up to $4.8 \mathrm{mg} / \mathrm{g}$ DCW) of $\beta$-carotene in YPD and YNB flask cultures, indicating a promisor future of the auxotrophic mutants IMUFRJ 50682 as a chassis for production of novel value-added chemicals.
\end{abstract}

\section{Introduction}

Yarrowia lipolytica is one of the most widely studied non-conventional oleaginous yeasts due to its valuable and diverse biosynthetic potential among different wild-type strains of industrial relevance [1]. It has emerged as an excellent cell factory for safe and high-level production of enzymes [2], unusual fatty acids [3], organic acids [4], polyalcohols [5], aromas [6], carotenoids [7] and bioemulsifiers [8] from different sources. Over the years, this yeast became a model in research on fungal dimorphic transition [9], recombinant protein secretion [10], microbial oil accumulation [11] and hydrophobic substrates degradation [12]. All these features have boosted the development of novel and efficient synthetic biology tools such as modular cloning systems $[13,14]$ and CRISPR/Cas9 technology that facilitate the manipulation of $Y$. lipolytica genome $[15,16]$.
In the CRISPR/Cas9 method, a single-guide RNA (sgRNA) complexed with a Cas9 nuclease can recognize a precise DNA sequence and generate a double-strand break (DSB) which is repaired by homologous recombination (HR) or non-homologous end-joining (NHEJ) mechanism [17]. The repair introduces small indels resulting in frameshift mutations that can cause disturbance of gene function, incorporation of an exogenous DNA or restoration of the original allele [18]. To date, several strategies CRISPR/Cas9-based have been developed for genetic improvement of microbial cell factories, since industrial biotechnology needs to meet the growing demands and expand the diversity of molecules that can be produced by non-model microorganisms [19]. Some wild-type strains with great advantage for specific bioproduction processes, such as Ashbya gossypii [20], Kluyveromyces marxianus [21], Aspergillus niger [22] and also Y. lipolytica have already been accurately engineered through CRISPR/Cas9 systems without limitations related

\footnotetext{
* Corresponding author at: Federal University of Rio de Janeiro, Department of Biochemistry, Institute of Chemistry, 541, 21941-909, Rio de Janeiro, RJ, Brazil.

** Corresponding author at: Federal University of Rio de Janeiro, Biochemical Engineering Department, School of Chemistry, 106, 21941-909, Rio de Janeiro, RJ, Brazil.

*** Corresponding author.

E-mail addresses: alice@eq.ufrj.br (M.A. Zarur Coelho), volcan@iq.ufrj.br (R.V. Almeida), jean-marc.nicaud@inrae.fr (J.-M. Nicaud).
} 
to selection markers [23].

Y. lipolytica IMUFRJ 50682 is a Brazilian wild-type strain isolated from an estuary in Guanabara Bay of Rio de Janeiro [24] with noticeable application in bioconversion and bioremediation [25]. This strain displayed peculiar surface characteristics related to the hydrophobic cell wall and high adhesion to non-polar solvents [26]. Y. lipolytica IMUFRJ 50682 is also able to secrete a particular bioemulsifier, a lipid-carbohydrate-protein complex, in the presence of glucose, glycerol, n-alkanes and oils [27,28]. Recently, IMUFRJ 50682 strain showed ability to consume isoprenoids and aromatic hydrocarbons as the group of naphthalenes and phenanthrene [29] and to be used as a low-cost biocatalyst for esterification reactions of fatty acids present in acid raw materials for biodiesel synthesis [30] and generation of commercial esters [31]. Although the industrial value of the IMUFRJ 50682 strain is remarkable, it has been little explored due to the lack of information about its genome.

Hence, we employed a combinatorial CRISPR/Cas9-mediated cleavage strategy for construction of a non-inverting auxotrophic mutants $Y$. lipolytica IMUFRJ 50682 through co-transformation of dual vectors harboring Cas9 nuclease and distinct combinations of sgRNAs targeting upstream and downstream sites of URA3 gene. Further, we determined the cloning efficiency of the auxotrophic derivatives with a fluorescent phenotype, introduced $\beta$-carotene biosynthetic pathway, compared the growth profile of the carotenoid recombinant producers and wild-type strain in both rich and synthetic media as well as quantified its production. Future applications of the auxotrophic mutants IMUFRJ 50682 were also discussed.

\section{Materials and methods}

\subsection{Microorganism and culture conditions}

Y. lipolytica IMUFRJ 50862 was cultivated at $28^{\circ} \mathrm{C}$ in rich YPD medium ( $1 \%$ yeast extract, $2 \%$ peptone, $2 \%$ glucose; w/v) for growth and synthetic YNB medium $(0.17 \%$ yeast nitrogen base without amino acids, $0.5 \% \mathrm{NH}_{4} \mathrm{Cl}, 2 \%$ glucose; $\mathrm{w} / \mathrm{v}$ and $50 \mathrm{mM}$ phosphate buffer $\mathrm{pH}$ 6.8) for screening of transformants. Alternatively, YPD medium was supplemented with $300 \mu \mathrm{g} \cdot \mathrm{mL}^{-1}$ hygromycin B (YPD-Hyg) (Corning), $500 \mu \mathrm{g} \cdot \mathrm{mL}^{-1}$ nourseothricin (YPD-Nat) (Werner BioAgents) or both antibiotics (YPD-Hyg-Nat) for selection of co-transformants and cured strains. Auxotrophic derivatives were tested in YNB medium added of $0.1 \%$ uracil (w/v; YNB-Ura) (Sigma-Aldrich). Escherichia coli DH5a were grown at $37^{\circ} \mathrm{C}$ in LB medium ( $1 \%$ tryptone, $0.5 \%$ yeast extract and $1 \% \mathrm{NaCl} ; \mathrm{w} / \mathrm{v}$ ) supplied with $100 \mu \mathrm{g} \cdot \mathrm{mL}^{-1}$ ampicillin (SigmaAldrich) for cloning and propagation of recombinant plasmids. When required, solid media were prepared by adding $1.5 \%$ agar $(\mathrm{w} / \mathrm{v})$ to liquid media. All cultures were maintained at $-80^{\circ} \mathrm{C}$ in glycerol stock.

\subsection{Assembly of CRISPR-Cas9 vectors}

Target sequences for URA3 deletion were predicted by the CRISPOR tool (http://crispor.tefor.net/) [32] based on Y. lipolytica W29 (Accession No. AJ306421.1). Two highest scoring 20 bp sequences targeting URA3transcriptional start site (UTSS) and transcriptional end site (UTES) with low number of predicted off-target and without protospacer adjacent motif (PAM) sequence were selected. sgRNAs were designed as complementary oligonucleotides, flanked by overhangs for plasmid assembly. All oligos were supplied by Eurofins Genomics and were listed in Supplementary File (Table S1).

CRISPR-Cas9 vectors were constructed by Golden Gate Assembly (GGA) [33]. The backbone plasmids were developed prior works [23]. Phosphorylated and annealed sgRNAs were diluted (ratio of 1:200) and then $2 \mu \mathrm{L}$ was mixed in one-step reaction with $100 \mathrm{ng}$ of acceptor plasmids, $10 \mathrm{U}$ of BsmBI, $3000 \mathrm{U}$ of T7 DNA ligase, $2 \mu \mathrm{L}$ of DNA ligase buffer and $\mathrm{ddH}_{2} \mathrm{O}$ up to $20 \mu \mathrm{L}$. sgRNA-UTSS1 and sgRNA-UTSS2 were separately added to the GGA_NATex_CrisprCas9-yl_RFP reaction, whereas sgRNA-UTES1 and sgRNA-UTES2 were added in the GGA_HPHex_CrisprCas9-yl_RFP mix. Thermal cycles were applied as follows: [55 ${ }^{\circ} \mathrm{C}$ for $5 \mathrm{~min}, 16^{\circ} \mathrm{C}$ for $5 \mathrm{~min}$ ] $\mathrm{x} 30,50^{\circ} \mathrm{C}$ for $5 \mathrm{~min}, 80^{\circ} \mathrm{C}$ for $5 \mathrm{~min}$, $15^{\circ} \mathrm{C} \infty$. The reaction mixture was used for chemically competent $E$. coli cells transformation and some ampicillin-resistant white colonies were screened for verification of assembled plasmids through BglII digestion. Restriction enzymes, ligases and kinases were obtained from New England Biolabs and performed in accordance with manufacturer's instructions. All plasmids used or constructed in this study were listed in Table S2.

\subsection{Construction of $Y$. lipolytica strains}

Correct CRISPR/Cas9 vectors were directly used to transform Y. lipolytica IMUFRJ 50682 by adapted lithium acetate method [34]. Briefly, about $500 \mathrm{ng}$ of each CRISPR-Cas9 vectors were cotransformed. CC_sgRNA-UTSS and CC_sgRNA-UTES plasmids were combined in the same transformation reaction for URA3 excision. The mixture was plated and incubated for 2-4 days. All transformations were performed in duplicate. Genotype verification of URA3 gene disruption were performed by colony PCR (GoTaq ${ }^{\circledast}$ Master Mix, Promega) using FwUTSS/Rv-UTES oligos (Table S1). PCR products were purified (NucleoSpin ${ }^{\circledR}$ PCR Clean-up kit, Macherey-Nagel) and then subjected to sequencing (Eurofins Genomics). Multiple alignment of the nucleotide sequences was performed by MAFFT online service (https://mafft.cbrc. jp/alignment/server/) [35].

Auxotrophic derivatives were transformed with NotI linearized GGVA-RedStarII plasmid [36] and Sfil digested GGVA-Carotenoid vector [33]. All colonies grown on YNB plate from the transformation with GGVA-RedStarII were cultivated in 96-well microplates containing YPD medium for $24 \mathrm{~h}$ in constant shaking and fluorescence was measured with excitation at $485 \mathrm{~nm}$ and emission at $528 \mathrm{~nm}$ (BioTek Synergy $^{\mathrm{TM}} \mathrm{Mx}$ ). The cloning efficiency was determined as the total colonies in which a fluorescent phenotype was observed by all colonies grown after transformation.

\subsection{Determination of growth profile}

Orange colonies from the transformation with GGVA-Carotenoid were grown in YPD medium at $200 \mathrm{rpm}$ overnight. Cultures were washed, adjusted to an initial $\mathrm{OD}_{750 \mathrm{~nm}}$ of 0.2 in 96 -well microplates containing fresh YPD and YNB medium and cultivated at $500 \mathrm{rpm}$ in a microplate shaker (Labstac LS122) for approximately $10 \mathrm{~h}$. Growth was measured as absorbance at $750 \mathrm{~nm}$ every $30 \mathrm{~min}$ (SpectraMax ${ }^{\circledR}$ M2e). All cultivations were done in duplicate and the maximal specific growth rate $\left(\mu_{\max }\right)$ were analyzed with one-way ANOVA.

\subsection{Extraction and quantification of $\beta$-carotene}

Intracellular $\beta$-carotene produced by recombinant IMUFRJ 50682 strains was extracted and measured photometrically at $455 \mathrm{~nm}$ (Thermo Scientific Genesys 10S) as published previously [7]. Then, cultures were inoculated with $\mathrm{OD}_{750 \mathrm{~nm}}$ of $0.1 \mathrm{in} 50 \mathrm{~mL}$ flasks containing $10 \mathrm{~mL}$ of YPD and YNB media. After grown at $200 \mathrm{rpm}$ for $96 \mathrm{~h}, 500 \mu \mathrm{L}$ of cultures were washed with ddH2O and $500 \mu \mathrm{L}$ of glass beads and $1 \mathrm{~mL}$ of extraction solution (ethyl acetate and $0.01 \%$ butyl hydroxyl toluene) were added. Cells were disrupted in a BeadBug cell homogenizer (Benchmark Scientific) using a program consisting in 4 cycles of $60 \mathrm{~s}$ at $4.000 \mathrm{rpm}$ in the dark, altering with ice incubation until the mixture were colorless. The extract was centrifuged, measured and then converted into concentrations through a standard curve using $\beta$-carotene (Sigma-Aldrich) as standard. For determination of dry cell weight (DCW), cells from a known volume were washed and dried at $100^{\circ} \mathrm{C}$. All cultures were performed in duplicate. 


\section{Results and discussion}

CRISPR/Cas9 technology is a powerful genome-editing tool and several methods based on Type II System have been developed recently [37]. In dual cleavage strategy, two independent sgRNAs, combined with Cas9 nuclease, induce DNA DSB in different regions which are repaired together by the NHEJ pathway, leading to complete loss of any sequence between target sites [38]. Thus, the present work investigated the efficiency of this strategy in removing all URA3 coding sequence in a promising wild-type strain, Y. lipolytica IMUFRJ 50p@ssw0rd

682 , to construct auxotrophic derivatives. URA3 gene encodes orotidine $5^{\prime}$-phosphate decarboxylase, an enzyme that catalyzes the conversion of orotidine $5^{\prime}$-monophosphate (OMP) to uridine $5^{\prime}$-monophosphate (UMP), the last step in pyrimidine biosynthesis [39]. URA3 deletion prevents yeast cells from growing in the absence of uracil and this auxotrophy phenotype still remains the best choice for a selective marker in recipient $Y$. lipolytica strains used in synthetic biology $[40,41]$.

To knock out native URA3, two pairs of 20 bp target sequences located in transcriptional initiation sites (sgRNA UTSS1: -79 to -59 bp; sgRNA UTSS2: -139 to $-119 \mathrm{bp}$ ) and termination regions (sgRNA UTSS1: +888 to +908 bp; sgRNA UTSS2: +1002 to +1022 bp) were predicted (Fig. 1A). Each target sequence was used in the construction of four CRISPR/Cas9 replicative vectors through GGA method. Acceptor plasmids GGA_NATex_CrisprCas9-yl_RFP and GGA_HPHex_CrisprCas9yl_RFP owned same construction (Fig. S1) with differences only in the target sequence and selection markers to allow us to identify which regions are more favorable for URA3 gene editing in $Y$. lipolytica
IMUFRJ 50682, since gene excision through dual DSB CRISPR/Cas9mediated requires synchronized cleavage of both sites during the same cell cycle [42]. CC_sgRNA-UTSS and CC_sgRNA-UTES constructed plasmids were mixed and co-transformed in IMUFRJ 50682 cells. Combination A was done with CC_sgRNA-UTSS1 and CC_sgRNA-UTES1 for excision of $973 \mathrm{bp}$. Combination B was performed with CC_sgRNAUTSS1 and CC_sgRNA-UTES2 for deletion of $1.073 \mathrm{bp}$. Combination C was between CC_sgRNA-UTSS2 and CC_sgRNA-UTES1 for loss of 1.052 bp. Combination D was accomplished with CC_sgRNA-UTSS2 and CC_sgRNA-UTES1 for disruption of $1.152 \mathrm{bp}$ (Fig. 1A).

After 4 days, a total of 101 hygromycin-B and nourseothricin-resistant colonies were observed in all combinations from biological duplicate: A (46 colonies), B (31 colonies), C ( 2 colonies) and D (22 colonies). Uracil auxotroph phenotype of the co-transformants was tested in YNB, YNB-Ura and YPD media (Fig. 1B). For combination A, 25 colonies were streaked on these media and $40 \%(10 / 25)$ was unable to grow on YNB. For combination B, 20 colonies were tested and only 5\% $(1 / 20)$ failed to grow in the selective medium. All co-transformants from combinations C and D were cultivated in YNB and were able to grow and, therefore, URA3 knock out did not occur. Each candidate from combinations A ( 10 colonies - C01, C02, C07, C08, C09, C10, C13, C59, C60 and C62) and B (1 colony - C23) was taken for verification by colony PCR and confirmation via DNA sequencing. No sequence was amplified from $\mathrm{C} 01, \mathrm{C} 09$ and $\mathrm{C} 62$ and the other eight candidates (C02, C07, C08, C10, C13, C23, C59, C60) showed amplified sequence $\leq 500$ bp confirming URA3 deletion, since amplified sequence from wild-type IMUFRJ 50682 had 1.221 bp length (Fig. 1C). From all sgRNA combinations, only in combinations A (UTSS1 and UTES1) and B (UTSS1 and

A

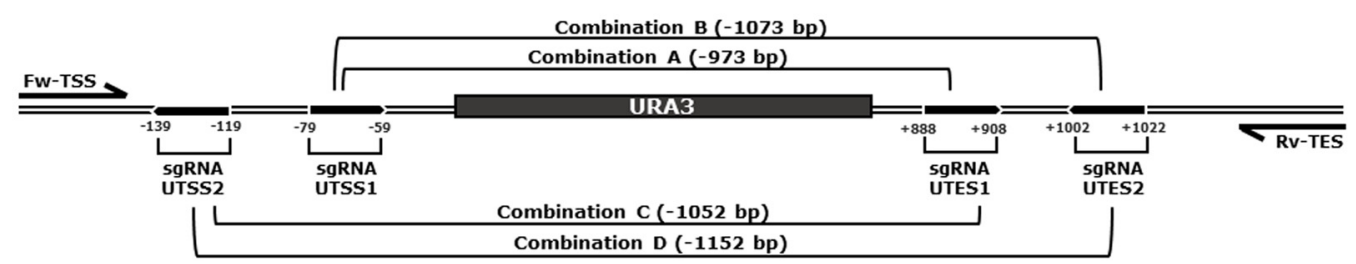

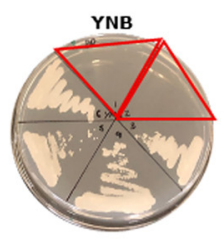

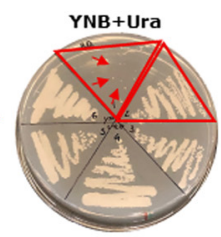

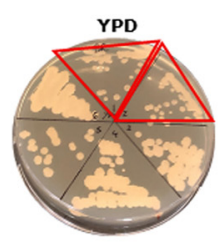

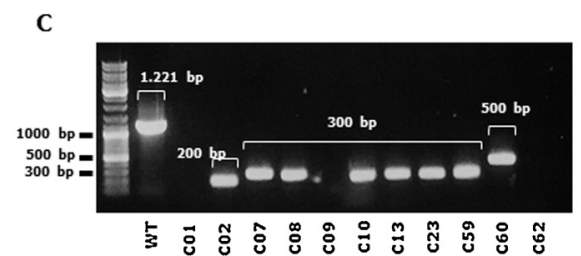

D

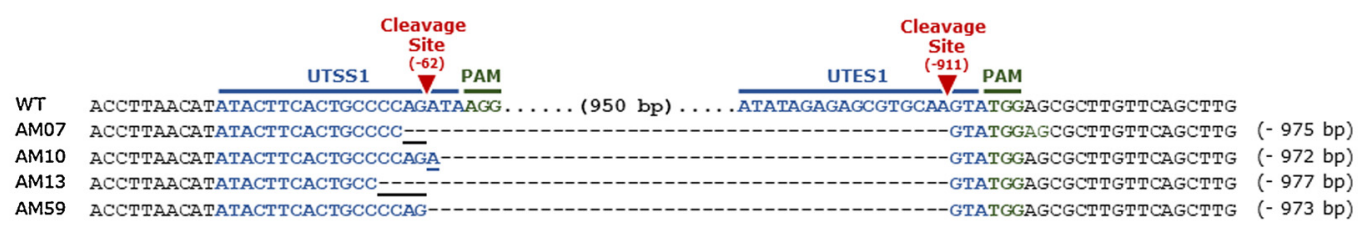

E

UTSS1 PAM

UTES1

PAM

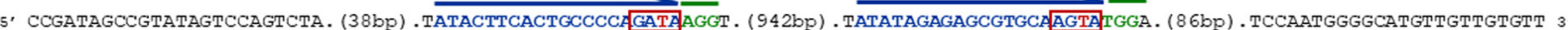

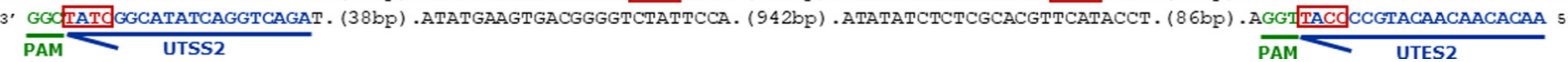

Fig. 1. URA3 deletion of Yarrowia lipolytica IMUFRJ 50682 by dual cleavage strategy. (A) Schematic representation of paired sgRNA combinations. Numbers in parentheses show fragment size to be lost through dual double-strand break. The position +1 indicate the start codon (ATG) and sgRNA locations are presented by their distance of coding sequence (in base pairs). (B) Screening of auxotrophic mutants IMUFRJ 50682 in YNB, YNB-Ura and YPD plates. Red arrows indicate presence of colonies. (C) Colony PCR of candidates unable to grow on synthetic YNB medium. Fragments with $\leq 500 \mathrm{bp}$ indicate URA3 excision. (D) Multiple alignment between amplified sequences $\left(5^{\prime} \rightarrow 3^{\prime}\right)$ of native URA3 and some auxotrophic mutants from combination A showing gene deletion. Letters in blue represent URA3 transcriptional start site 1 (UTSS1) and URA3 transcriptional end site 1 (UTES1), letters in green exhibit protospacer adjacent motif (PAM) sequence, red arrowheads show predicted cleavage site and indels are underlined. WT: wild-type. C01-62: candidates 01-62. AM07-59: auxotrophic mutants 07-59. (E) PAMproximal bases located in sgRNAs. Letters in green represent PAM sequence, letters in blue indicate sgRNAs sequence, blue arrowheads show sgRNAs direction and letters in red highlight unfavorable bases for Cas9 cleavage (For interpretation of the references to colour in this figure legend, the reader is referred to the web version of this article). 
Table 1

Efficiency of URA3 gene deletion by dual cleavage strategy using different sgRNA combinations. Numbers in parentheses represent screened colonies from biological duplicate. Deletion efficiency is presented as the percentage of positive colonies in genotypic confirmation by the total of uracil auxotrophy phenotype colonies.

\begin{tabular}{lllll}
\hline $\begin{array}{l}\text { Combinations } \\
\text { of sgRNAs }\end{array}$ & $\begin{array}{l}\text { Total } \\
\text { colonies }\end{array}$ & $\begin{array}{l}\text { Phenotype of } \\
\text { auxotrophy }\end{array}$ & $\begin{array}{l}\text { Genotype } \\
\text { confirmation }\end{array}$ & $\begin{array}{l}\text { Deletion } \\
\text { efficiency (\%) }\end{array}$ \\
\hline A (UTSS1 + UTES1) & 46 & $10(25)$ & $7(10)$ & 28 \\
B (UTSS1 + UTES2) & 31 & $1(20)$ & $1(1)$ & 5 \\
C (UTSS2 + UTES1) & 2 & $0(2)$ & - & - \\
D (UTSS2 + UTES2) & 22 & $0(22)$ & - & - \\
\hline
\end{tabular}

UTES2) were identified ura3s mutants IMUFRJ 50682 with deletion efficiency of $28 \%(7 / 25)$ and 5\% (1/20), respectively (Table 1$)$. Multiple alignment between amplified sequences of native URA3 gene and some auxotrophic mutants from combination A, now named AM07, AM10, AM13 and AM59, showed precise NHEJ repair only in AM59. NHEJ is an error-prone process often associated with small insertions and deletions at the DSB site which regularly occurs 3-4 bp upstream of the PAM sequence [43]. In the UTSS1 regions of AM07, AM10 and AM13 sequences were observed indels: AM07 had deletion of $2 \mathrm{bp}$, AM10 received addition of $1 \mathrm{bp}$ and AM13 lost $4 \mathrm{bp}$; totaling a loss of 975, 972 and 977 bp, respectively (Fig. 1D).

The choice of target sequences closer to URA3 start (-59 bp) and stop ( $+888 \mathrm{bp}$ ) codon proved to be more favorable for DNA cutting in IMUFRJ 50682 strain, since combinations A and B led to the excision of all gene. Although sgRNAs were designed based on W29 strain, all of them perfectly matched the recognition site in IMUFRJ 50682 strain (Fig. S2), even no auxotrophic mutants have been generated from combinations C and D. According to Graf and colleagues [44], the specificity of a sgRNA depends on its target sequence and recognition site with $\geq 80 \%$ or $\leq 35 \%$ of guanine-cytosine (GC) content has been shown poorly effective. Overall, Cas9 nuclease has demonstrated preferential binding in sgRNAs with $\mathrm{G}$ in the 4 bp directly upstream to the PAM sequence, while thymine $(\mathrm{T})$ and $\mathrm{C}$ were unfavorable $[45,46]$. The low URA3 deletion frequencies in our study may be due to the presence of such adverse bases in short sequence motifs at the $3^{\prime}$ end of sgRNAs. The UTSS 2 and UTES2 are reverse sgRNAs and possess $5^{\prime}$-CTAT- $3^{\prime}$ and $5^{\prime}-$ CCAT- $3^{\prime}$ sequences, respectively, which are rich in TC bases. The UTSS1 and UTES1 sgRNAs have 5'-GATA-3' and 5'-AGTA-3' sequences, respectively, with only one $\mathrm{T}$, suggesting correlation between pyrimidine bases near the PAM sequence and Cas9 nuclease performance (Fig. 1E). In addition, analysis in the sgRNA sequences by RNA Structure and RNAfold WEB SERVER platforms indicated the possibility of secondary structure formation in UTSS2, which would prevent the recognition of the target DNA and its cleavage. A substantial variation in the cleavage efficiency in accordance with different genomic targets may be related not only to the inherent quality of the interaction between the sgRNA and the target sequence, but also to the general chromatin accessibility by the Cas 9 nuclease, even within the same cell type [47]. The combinatorial CRISPR/Cas9-mediated cleavage strategy has proven to be effective for engineering strains without genetic information.

The ura3 $\Delta$ mutants IMUFRJ 50682 were sequentially cultivated in YNB-Ura medium by three rounds for plasmid curing, purified in YPD plates and verified in YPD-Hyg and YPD-Nat media. Then, AM07, AM10, AM13, AM23 and AM59 auxotrophic mutants were transformed with GGVA-RedStarII plasmid, which contains RedStarII expression cassette driven by pTEF and URA3 gene as selective marker [48], to constitutively express the red fluorescent protein as a gene reporter for verification of cloning efficiency. After 2 days, grown colonies were analyzed by fluorescence phenotype. The cloning rates were satisfactory of 82.6 (19/23), 91.6 (33/36), 100.0 (11/11), 80.0 (4/5) and 89.5 $\%(17 / 19)$ for AM07, AM10, AM13, AM23 and AM59 mutants, respectively, and motivated us to investigate the biotechnological potential of this strain.

In order to verify the applicability of $u r a 3 \Delta$ mutants IMUFRJ 50,682 as a chassis for production of non-native value-added compounds, auxotrophic mutants AM07, AM10, AM13, AM23 and AM59 were engineered for $\beta$-carotene synthesis. GGVA-Carotenoid plasmid contains the heterologous genes carB and carRP from Mucor circinelloides and native gene GGS1 under the control of constitutive promoter pTEF, for expression of phytoene dehydrogenase, phytoene synthase and geranylgeranyl diphosphate synthase, respectively, and URA3 gene as auxotrophic marker [33]. Three carotenoid recombinant producers, CRP13, CRP23 and CRP59, were selected according to intense orange colour-associated phenotype (Fig. 2A) and growth profile and specific growth rates of them were compared with Brazilian wild-type strain in rich and synthetic media. Throughout cultivation in rich medium, wildtype strain and CRP13, CRP23 and CRP59 recombinants displayed a very similar growth pattern and statistical analysis indicated no significant differences in the $\mu_{\max }$ ( $\mathrm{p}>0.05$ ). The IMUFRJ 50682 strain was capable of growing with a $\mu_{\max }$ of $0.38 \mathrm{~h}^{-1}$, whereas CRP13, CRP23 and CRP59 recombinants achieved a $\mu_{\max }$ of $0.34,0.34$ and 0.33 ,

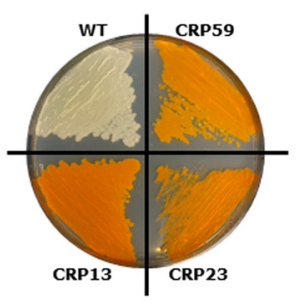

B

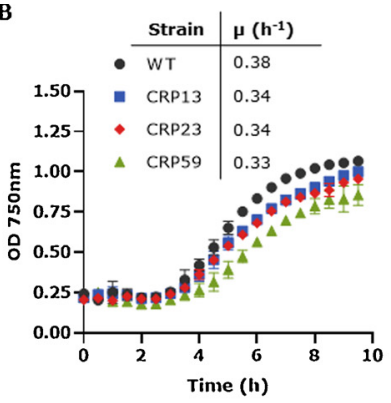

C

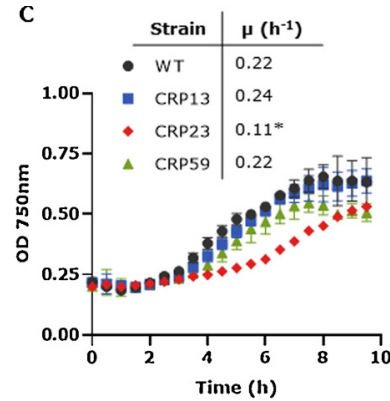

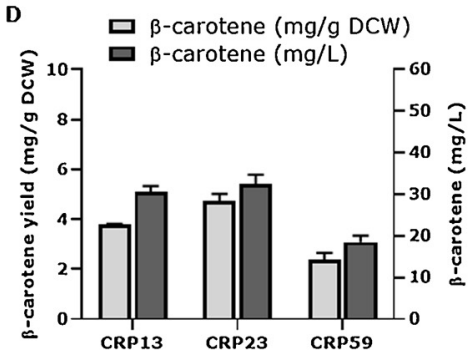

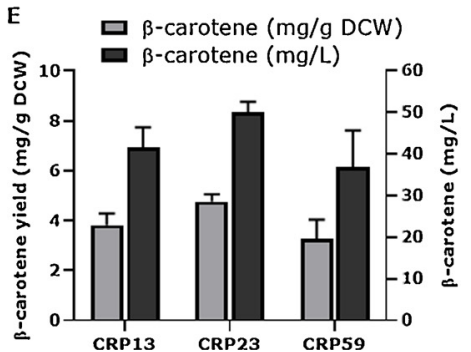

Fig. 2. Yarrowia lipolytica IMUFRJ 50682 engineering for $\beta$-carotene synthesis. (A) Carotenoid recombinant producers CRP13, CRP23 and CRP59 showed orange colour during cultivation in liquid media. (B) Growth profile and specific growth rates of wild-type and auxotrophic mutants in rich YPD and (C) synthetic YNB media. (D) $\beta$-carotene production in YPD and (E) YNB shake-flask cultures. Data are average of two independent cultivations.

*Indicate significant differences between specific growth rates of wild-type and carotenoid producers ( $p>0.05)$. WT: wild-type 
respectively (Fig. 2B). In synthetic medium, the $\mu_{\max }$ of wild-type strain and CRP13, CRP23 and CRP59 recombinants decreased sightly to 0.22 , $0.24,0.11$ and $0.22 \mathrm{~h}^{-1}$, respectively, and differed significantly between CRP23 and the other strains ( $<<0.05)$ (Fig. 2C). The increased metabolic load by overexpression of GGS1 gene and heterologous expression of $c a r B$ and carRP genes did not reduced growth capacity of CRP13 and CRP59 recombinants, indicating that the introduction of a new metabolic pathway was efficiently adapted by mutants IMUFRJ 50682.

Interestingly, CRP23 strain was able to synthetized $32.4(4.7 \mathrm{mg} / \mathrm{g}$ DCW $)$ and $50.1 \mathrm{mg} / \mathrm{L}(4.8 \mathrm{mg} / \mathrm{DCW})$ of $\beta$-carotene in YPD and YNB shake-flask cultures, respectively, while the final $\beta$-carotene titer of the CRP13 and CRP59 strains reached 30.5 and $41.6 \mathrm{mg} / \mathrm{L}(3.8 \mathrm{mg} / \mathrm{g}$ DCW) and $18.5(2.4 \mathrm{mg} / \mathrm{g}$ DCW $)$ and $36.8 \mathrm{mg} / \mathrm{L}(3.3 \mathrm{mg} / \mathrm{g}$ DCW $)$ in both media, respectively (Fig. 2D and E). The use of mutants IMUFRJ 50682 as a parental strain led to a higher $\beta$-carotene production than other backgrounds, such as Po1d (3.4 mg/g DCW) [23] and CIBTS1176 $(2.22 \mathrm{mg} / \mathrm{g}$ DCW) [49] strains which were engineered with the same genes and cultivated in similar conditions. These data reveal the metabolic potential of IMUFRJ 50682 strain to be explored.

As $Y$. lipolytica displays heterogeneity inter wild-type strains related to different genetics and metabolic patterns, the construction of auxotrophic derivatives from IMUFRJ 50682 will expand the range of applications for this promising strain. The expectation around $Y$. lipolytica IMUFRJ 50682 is due to its industrially relevant potential in the fields of biocatalysis, bioconversion and bioremediation. Previous studies from our group have demonstrated that this strain exhibits robust metabolism and it is able to degrade alternative substrates, such as compounds from poly(ethylene terephthalate) (PET) production chain, and convert some of them into value-added products with additional sustainability benefits and biotechnological processes economics [50]. As soon, we developed for the first time non-reverting auxotrophic mutants from $Y$. lipolytica IMUFRJ 50682 and evidenced their ability to produce new compounds of biotechnological interest.

\section{Conclusion}

The combinatorial dual CRISPR/Cas9 cleavage strategy proved to be useful and efficient for whole URA3 gene excision in wild-type strain $Y$. lipolytica IMUFRJ 50682. Auxotrophic mutants demonstrated high cloning frequency by introduction of fluorescent phenotype and were able to synthesize $\beta$-carotene efficiently through the expression of homologous and heterologous genes with a similar growth profile compared with the wild-type which motivates us to explore its genetic and physiology in future research.

\section{Author agreement}

I, the Corresponding Author, declare that this manuscript contains original, unpublished results and confirm that it has been read and approved by all the authors.

I understand that the Corresponding Author is the sole contact for the Editorial process and is responsible for communicating with the other authors about progress, submissions of revisions and final approval of proofs.

\section{Funding information}

This work was financed by grants from Brazilian Federal Agency CAPES (Proc. 88881.188542/2018-01) and French Nacional Agency INRAE.

\section{CRediT authorship contribution statement}

Camilla Pires de Souza: Conceptualization, Methodology, Writing original draft, Writing - review \& editing. Bernardo Dias Ribeiro:
Writing - review \& editing, Supervision. Maria Alice Zarur Coelho: Writing - review \& editing, Resources, Supervision. Rodrigo Volcan Almeida: Writing - review \& editing, Resources, Supervision, Funding acquisition. Jean Marc Nicaud: Writing - review \& editing, Resources, Supervision, Funding acquisition.

\section{Declaration of Competing Interest}

The authors declare no conflict of interest.

\section{Acknowledgements}

We are grateful for the National Research Institute for Agriculture, Food and Environment (INRAE), Federal University of Rio de Janeiro (UFRJ) and Higher Education Improvement Coordination (CAPES).

\section{Appendix A. Supplementary data}

Supplementary material related to this article can be found, in the online version, at doi:https://doi.org/10.1016/j.enzmictec.2020. 109621.

\section{References}

[1] M. Egermeier, H. Russmayer, M. Sauer, H. Marx, Metabolic flexibility of Yarrowia lipolyticagrowing on glycerol, Front. Microbiol. 8 (2017) 1-9, https://doi.org/10. 3389/fmicb.2017.00049.

[2] R. Dulermo, F. Brunel, T. Dulermo, R. Ledesma-Amaro, J. Vion, M. Trassaert, S. Thomas, J.M. Nicaud, C. Leplat, Using a vector pool containing variable-strength promoters to optimize protein production in Yarrowia lipolytica, Microb. Cell Fact. 16 (2017) 1-11, https://doi.org/10.1186/s12934-017-0647-3.

[3] Y.K. Park, T. Dulermo, R. Ledesma-Amaro, J.M. Nicaud, Optimization of odd chain fatty acid production by Yarrowia lipolytica, Biotechnol. Biofuels 11 (2018) 1-12, https://doi.org/10.1186/s13068-018-1154-4.

[4] A. Rywińska, W. Rymowicz, High-yield production of citric acid by Yarrowia lipolyticaon glycerol in repeated-batch bioreactors, J. Ind. Microbiol. Biotechnol. 37 (2010) 431-435, https://doi.org/10.1007/s10295-009-0687-8.

[5] F. Carly, M. Vandermies, S. Telek, S. Steels, S. Thomas, J.M. Nicaud, P. Fickers, Enhancing erythritol productivity in Yarrowia lipolytica using metabolic engineering, Metab. Eng. 42 (2017) 19-24, https://doi.org/10.1016/j.ymben.2017. 05.002.

[6] E. Celińska, P. Kubiak, W. Białas, M. Dziadas, W. Grajek, Yarrowia lipolytica: the novel and promising 2-phenylethanol producer, J. Ind. Microbiol. Biotechnol. 40 (2013) 389-392, https://doi.org/10.1007/s10295-013-1240-3.

[7] M. Larroude, E. Celinska, A. Back, S. Thomas, J.M. Nicaud, R. Ledesma-Amaro, A synthetic biology approach to transform Yarrowia lipolytica into a competitive biotechnological producer of $\beta$-carotene, Biotechnol. Bioeng. 115 (2018) 464-472, https://doi.org/10.1002/bit.26473.

[8] F.A.S.D. Souza, A.A. Salgueiro, C.D.C. Albuquerque, Production of bioemulsifiers by Yarrowia lipolytica in sea water using diesel oil as the carbon source, Brazilian J. Chem. Eng. 29 (2012) 61-67, https://doi.org/10.1590/S010466322012000100007.

[9] A. Martinez-Vazquez, A. Gonzalez-Hernandez, Á. Domínguez, R. Rachubinski, M. Riquelme, P. Cuellar-Mata, J.C.T. Guzman, Identification of the transcription factor Znc1p, which regulates the yeast-to-Hypha transition in the dimorphic yeast Yarrowia lipolytica, PLoS One 8 (2013), https://doi.org/10.1371/journal.pone. 0066790.

[10] J.M. Nicaud, C. Madzak, P. Van Den Broek, C. Gysler, P. Duboc, P. Niederberger, C. Gaillardin, Protein expression and secretion in the yeast Yarrowia lipolytica, FEMS Yeast Res. 2 (2002) 371-379, https://doi.org/10.1016/S1567-1356(02)00082-X.

[11] A. Beopoulos, J. Cescut, R. Haddouche, J.L. Uribelarrea, C. Molina-Jouve, J.M. Nicaud, Yarrowia lipolytica as a model for bio-oil production, Prog. Lipid Res. 48 (2009) 375-387, https://doi.org/10.1016/j.plipres.2009.08.005.

[12] J.S. Sabirova, R. Haddouche, I.N. Van Bogaert, F. Mulaa, W. Verstraete, K.N. Timmis, C. Schmidt-Dannert, J.M. Nicaud, W. Soetaert, The "LipoYeasts" project: Using the oleaginous yeast Yarrowia lipolytica in combination with specific bacterial genes for the bioconversion of lipids, fats and oils into high-value products, Microb. Biotechnol. 4 (2011) 47-54, https://doi.org/10.1111/j.1751-7915. 2010.00187.x.

[13] L. Wong, J. Engel, E. Jin, B. Holdridge, P. Xu, YaliBricks, a versatile genetic toolkit for streamlined and rapid pathway engineering in Yarrowia lipolytica, Metab. Eng. Commun. 5 (2017) 68-77, https://doi.org/10.1016/j.meteno.2017.09.001.

[14] M. Egermeier, M. Sauer, H. Marx, Golden Gate-based metabolic engineering strategy for wild-type strains of Yarrowia lipolytica, FEMS Microbiol. Lett. 366 (2019), https://doi.org/10.1093/femsle/fnz022.

[15] C. Schwartz, M. Shabbir-Hussain, K. Frogue, M. Blenner, I. Wheeldon, Standardized markerless gene integration for pathway engineering in Yarrowia lipolytica, ACS Synth. Biol. 6 (2017) 402-409, https://doi.org/10.1021/acssynbio.6b00285. 
[16] C. Holkenbrink, M.I. Dam, K.R. Kildegaard, J. Beder, J. Dahlin, D. Doménech Belda, I. Borodina, EasyCloneYALI: CRISPR/Cas9-based synthetic toolbox for engineering of the yeast Yarrowia lipolytica, Biotechnol. J. 13 (2018) 1-8, https://doi.org/10. 1002/biot.201700543.

[17] S. Gao, Y. Tong, Z. Wen, L. Zhu, M. Ge, D. Chen, Y. Jiang, S. Yang, Multiplex gene editing of the Yarrowia lipolytica genome using the CRISPR-Cas9 system, J. Ind. Microbiol. Biotechnol. 43 (2016) 1085-1093, https://doi.org/10.1007/s10295016-1789-8.

[18] M. Kosicki, S.S. Rajan, F.C. Lorenzetti, H.H. Wandall, Y. Narimatsu, E. Metzakopian, E.P. Bennett, Dynamics of indel profiles induced by various CRISPR/Cas9 delivery methods, Elsevier Inc. (2017), https://doi.org/10.1016/bs.pmbts.2017.09.003.

[19] P.D. Donohoue, R. Barrangou, A.P. May, Advances in industrial biotechnology using CRISPR-Cas systems, Trends Biotechnol. (2018), https://doi.org/10.1016/j.tibtech. 2017.07.007.

[20] A. Jiménez, G. Muñoz-Fernández, R. Ledesma-Amaro, R.M. Buey, J.L. Revuelta, One-vector CRISPR/Cas9 genome engineering of the industrial fungus Ashbya gossypii, Microb. Biotechnol. 12 (2019) 1293-1301, https://doi.org/10.1111/17517915.13425.

[21] P. Cernak, R. Estrela, S. Poddar, J.M. Skerker, Y.F. Cheng, A.K. Carlson, B. Chen, V.M. Glynn, M. Furlan, O.W. Ryan, M.K. Donnelly, A.P. Arkin, J.W. Taylor, J.H.D. Cate, Engineering Kluyveromyces marxianusas a robust synthetic biology platform host, MBio. (2018), https://doi.org/10.1128/mBio.01410-18.

[22] C.S. Nødvig, J.B. Nielsen, M.E. Kogle, U.H. Mortensen, A CRISPR-Cas9 system for genetic engineering of filamentous fungi, PLoS One (2015), https://doi.org/10. 1371/journal.pone.0133085.

[23] M. Larroude, H. Trabelsi, J.-M. Nicaud, T. Rossignol, A set of Yarrowia lipolyticaCRISPR/Cas9 vectors for exploiting wild-type strain diversity, Biotechnol. Lett. 4 (2020), https://doi.org/10.1007/s10529-020-02805-4.

[24] A.N. Hagler, L.C. Mendonça-Hagler, Yeasts from marine and estuarine waters with different levels of pollution in the state of rio de janeiro, Brazil., Appl. Environ. Microbiol. 41 (1981) 173-178 http://www.ncbi.nlm.nih.gov/pubmed/16345683\% 0Ahttp://www.pubmedcentral.nih.gov/articlerender.fcgi?artid=PMC243658.

[25] M.a Z. Coelho, P.F.F. Amaral, I. Belo, Yarrowia lipolytica: an industrial workhorse, Appl. Microbiol. Microb. Biotechnol. (2010) 930-944.

[26] P.F.F. Amaral, M. Lehocky, A.M.V. Barros-Timmons, M.H.M. Rocha-Leão, M.A.Z. Coelho, J.A.P. Coutinho, Cell surface characterization of Yarrowia lipolytica IMUFRJ 50682, Yeast. (2006), https://doi.org/10.1002/yea.1405.

[27] P.F.F. Amaral, J.M. da Silva, M. Lehocky, A.M.V. Barros-Timmons, M.A.Z. Coelho, I.M. Marrucho, J.A.P. Coutinho, Production and characterization of a bioemulsifier from Yarrowia lipolytica, Process Biochem. 41 (2006) 1894-1898, https://doi.org/ 10.1016/j.procbio.2006.03.029.

[28] M.A. Zarur Coelho, G.C. Fontes, P.F. Fonseca Amaral, M. Nele, Factorial design to optimize biosurfactant production by Yarrowia lipolytica, J. Biomed. Biotechnol. 2010 (2010), https://doi.org/10.1155/2010/821306.

[29] F.F. Martins, T.F. Ferreira, D.A. Azevedo, M.A.Z. Coelho, Evaluation of crude oil degradation by Yarrowia lipolytica, Chem. Eng. Trans. 27 (2012) 223-228, https:// doi.org/10.3303/CET1227038.

[30] J.R. da Silva, C.E.C. de Souza, E. Valoni, A.M. de Castro, M.A.Z. Coelho, B.D. Ribeiro, C.A. Henriques, M.A.P. Langone, Biocatalytic esterification of fatty acids using a low-cost fermented solid from solid-state fermentation with Yarrowia lipolytica, 3 Biotech 9 (2019) 0, https://doi.org/10.1007/s13205-018-1550-2.

[31] C.E.C. de Souza, B.D. Ribeiro, M.A.Z. Coelho, Characterization and application of yarrowia lipolytica lipase obtained by solid-state fermentation in the synthesis of different esters used in the food industry, Appl. Biochem. Biotechnol. (2019), https://doi.org/10.1007/s12010-019-03047-5.

[32] M. Haeussler, K. Schönig, H. Eckert, A. Eschstruth, J. Mianné, J.B. Renaud, S. Schneider-Maunoury, A. Shkumatava, L. Teboul, J. Kent, J.S. Joly, J.P. Concordet, Evaluation of off-target and on-target scoring algorithms and integration into the guide RNA selection tool CRISPOR, Genome Biol. (2016), https://doi.org/10.1186/s13059-016-1012-2.

[33] E. Celińska, R. Ledesma-Amaro, M. Larroude, T. Rossignol, C. Pauthenier, J.M. Nicaud, Golden Gate Assembly system dedicated to complex pathway manipulation in Yarrowia lipolytica, Microb. Biotechnol. 10 (2017) 450-455, https:// doi.org/10.1111/1751-7915.12605.

[34] D.C. Chen, J.M. Beckerich, C. Gaillardin, One-step transformation of the dimorphic yeast Yarrowia lipolytica, Appl. Microbiol. Biotechnol. (1997), https://doi.org/10. 1007/s002530051043.

[35] K. Katoh, J. Rozewicki, K.D. Yamada, MAFFT online service: multiple sequence alignment, interactive sequence choice and visualization, Brief. Bioinform. (2019), https://doi.org/10.1093/bib/bbx108.

[36] Y.K. Park, P. Korpys, M. Kubiak, E. Celinska, P. Soudier, P. Trébulle, M. Larroude, T. Rossignol, J.M. Nicaud, Engineering the architecture of erythritol-inducible promoters for regulated and enhanced gene expression in Yarrowia lipolytica, FEMS Yeast Res. (2019), https://doi.org/10.1093/femsyr/foy105.

[37] F. Darvishi, M. Ariana, E.R. Marella, I. Borodina, Advances in synthetic biology of oleaginous yeast Yarrowia lipolytica for producing non-native chemicals, Appl. Microbiol. Biotechnol. 102 (2018) 5925-5938, https://doi.org/10.1007/s00253018-9099-x.

[38] F. Adikusuma, C. Pfitzner, P.Q. Thomas, Versatile single-step-assembly CRISPR/ Cas9 vectors for dual gRNA expression, PLoS One 12 (2017) 1-11, https://doi.org/ 10.1371/journal.pone.0187236.

[39] E. Alani, L. Cao, N. Kleckner, A method for gene disruption that allows repeated use of, Genetics. 116 (1987) 541-545.

[40] C. Madzak, C. Gaillardin, J.M. Beckerich, Heterologous protein expression and secretion in the non-conventional yeast Yarrowia lipolytica: a review, J. Biotechnol. 109 (2004) 63-81, https://doi.org/10.1016/j.jbiotec.2003.10.027.

[41] M. Larroude, T. Rossignol, J.M. Nicaud, R. Ledesma-Amaro, Synthetic biology tools for engineering Yarrowia lipolytica, Biotechnol. Adv. 36 (2018) 2150-2164, https:// doi.org/10.1016/j.biotechadv.2018.10.004.

[42] D. Gao, S. Smith, M. Spagnuolo, G. Rodriguez, M. Blenner, Dual CRISPR-Cas9 cleavage mediated gene excision and targeted integration in Yarrowia lipolytica, Biotechnol. J. (2018), https://doi.org/10.1002/biot.201700590.

[43] J.E. Garneau, M.È. Dupuis, M. Villion, D.A. Romero, R. Barrangou, P. Boyaval, C. Fremaux, P. Horvath, A.H. Magadán, S. Moineau, The CRISPR/cas bacterial immune system cleaves bacteriophage and plasmid DNA, Nature. 468 (2010) 67-71, https://doi.org/10.1038/nature09523.

[44] R. Graf, X. Li, V.T. Chu, K. Rajewsky, sgRNA sequence motifs blocking efficient CRISPR/Cas9-Mediated gene editing, Cell Rep. 26 (2019) 1098-1103, https://doi. org/10.1016/j.celrep.2019.01.024 e3.

[45] T. Wang, J.J. Wei, D.M. Sabatini, E.S. Lander, Genetic screens in human cells using the CRISPR-Cas9 system, BMJ Support, Palliat. Care (2012), https://doi.org/10. 1136/bmjspcare-2011-000063.

[46] J.G. Doench, E. Hartenian, D.B. Graham, Z. Tothova, M. Hegde, I. Smith, M. Sullender, B.L. Ebert, R.J. Xavier, D.E. Root, Rational design of highly active sgRNAs for CRISPR-Cas9-mediated gene inactivation, Nat. Biotechnol. (2014), https://doi.org/10.1038/nbt.3026.

[47] R.M. Yarrington, S. Verma, S. Schwartz, J.K. Trautman, D. Carroll, Nucleosomes inhibit target cleavage by CRISPR-Cas9 in vivo, Proc. Natl. Acad. Sci. U. S. A. (2018), https://doi.org/10.1073/pnas.1810062115.

[48] M. Larroude, Y.K. Park, P. Soudier, M. Kubiak, J.M. Nicaud, T. Rossignol, A modular Golden Gate toolkit for Yarrowia lipolytica synthetic biology, Microb. Biotechnol. (2019), https://doi.org/10.1111/1751-7915.13427.

[49] S. Gao, L. Han, L. Zhu, M. Ge, S. Yang, Y. Jiang, D. Chen, One-step integration of multiple genes into the oleaginous yeast Yarrowia lipolytica, Biotechnol. Lett. (2014), https://doi.org/10.1007/s10529-014-1634-y. 